\title{
Single domain integral transform analysis of natural convection in cavities partially filled with heat generating porous medium
}

\section{Kleber Marques Lisboa, Jian Su \& Renato Machado Cotta}

To cite this article: Kleber Marques Lisboa, Jian Su \& Renato Machado Cotta (2018) Single domain integral transform analysis of natural convection in cavities partially filled with heat generating porous medium, Numerical Heat Transfer, Part A: Applications, 74:3, 1068-1086, DOI: 10.1080/10407782.2018.1511141

To link to this article: https://doi.org/10.1080/10407782.2018.1511141

\section{Published online: 08 Oct 2018.}

\section{Submit your article to this journal $\square$}

山 Article views: 39

View Crossmark data ¿ 


\title{
Single domain integral transform analysis of natural convection in cavities partially filled with heat generating porous medium
}

\author{
Kleber Marques Lisboa ${ }^{\mathrm{a}}$, Jian $\mathrm{Su}^{\mathrm{b}}$, and Renato Machado Cotta ${ }^{\mathrm{a} *}$ \\ aLaboratory of Nano- and Microfluidics and Microsystems, LabMEMS, Mechanical Engineering Department, \\ POLI \& COPPE, Interdisciplinary Nucleus for Social Development-NIDES/CT, Universidade Federal do Rio de \\ Janeiro, Rio de Janeiro, Brazil; ' ${ }^{\mathrm{b}}$ Nuclear Engineering Department and Nanoengineering Department, COPPE, \\ Universidade Federal do Rio de Janeiro, Rio de Janeiro, Brazil
}

\begin{abstract}
An analysis of natural convection within a rectangular cavity partially filled with a heat-generating porous medium is carried out through the Generalized Integral Transform Technique (GITT), in which the laminar flow and energy equations are solved with automatic error control. A single domain reformulation strategy is adopted to rewrite the governing equations within the fluid and the porous medium as a single heterogeneous medium formulation, with spatially variable physical properties and source terms that account for the abrupt transition of the two regions. This fundamental study is motivated by the analysis of wet storage of spent nuclear fuel elements with passive cooling of the pool and physical conclusions are drawn from the hybrid numerical-analytical solution. Increases in the Rayleigh number with constant internal heat generation are found to lower the maximum temperature within the cavity. Moreover, decreasing the aspect ratio also has positive effects on the cooling of the cavity.
\end{abstract}

\section{ARTICLE HISTORY}

Received 27 April 2018

Accepted 8 August 2018

\section{Introduction}

Heat and fluid flow phenomena in partially porous heterogeneous media still pose challenges in both mathematical modeling and solution methodology towards their accurate simulation $[1,2]$. Reliable models for both the bulk of the porous medium and its interface with a fluid layer must be employed. The most straightforward model is considered to be the use of the classical Darcy model and the Navier-Stokes equations in the porous and fluid regions, respectively, coupled by a semi-empirical velocity jump boundary condition at the interface [3-5]. Another approach involves the inclusion of the Brinkman correction [6] into the Darcy model using the volume averaging technique [7-9]. The presence of a second order term allows for the employment of continuity boundary conditions for both stress and velocity vectors at the interface between porous and fluid regions. The equivalency between the two formulations has been reported previously in the literature, provided that the adopted slip coefficient be proportional to the square root of the effective viscosity [10].

CONTACT Renato Machado Cotta cotta@mecanica.coppe.ufrj.br E Laboratory of Nano- and Microfluidics and Microsystems, LabMEMS, Mechanical Engineering Department, POLI \& COPPE, Interdisciplinary Nucleus for Social Development-NIDES/CT, Universidade Federal do Rio de Janeiro, Cidade Universitária, Cx. Postal 68503, Rio de Janeiro, RJ, CEP 21941-972, Brazil.

*Present Address: Mechanical Engineering Department, University College London, UCL, London, UK.

Color versions of one or more of the figures in the article can be found online at www.tandfonline.com/unht.

(C) 2018 Taylor \& Francis Group, LLC 


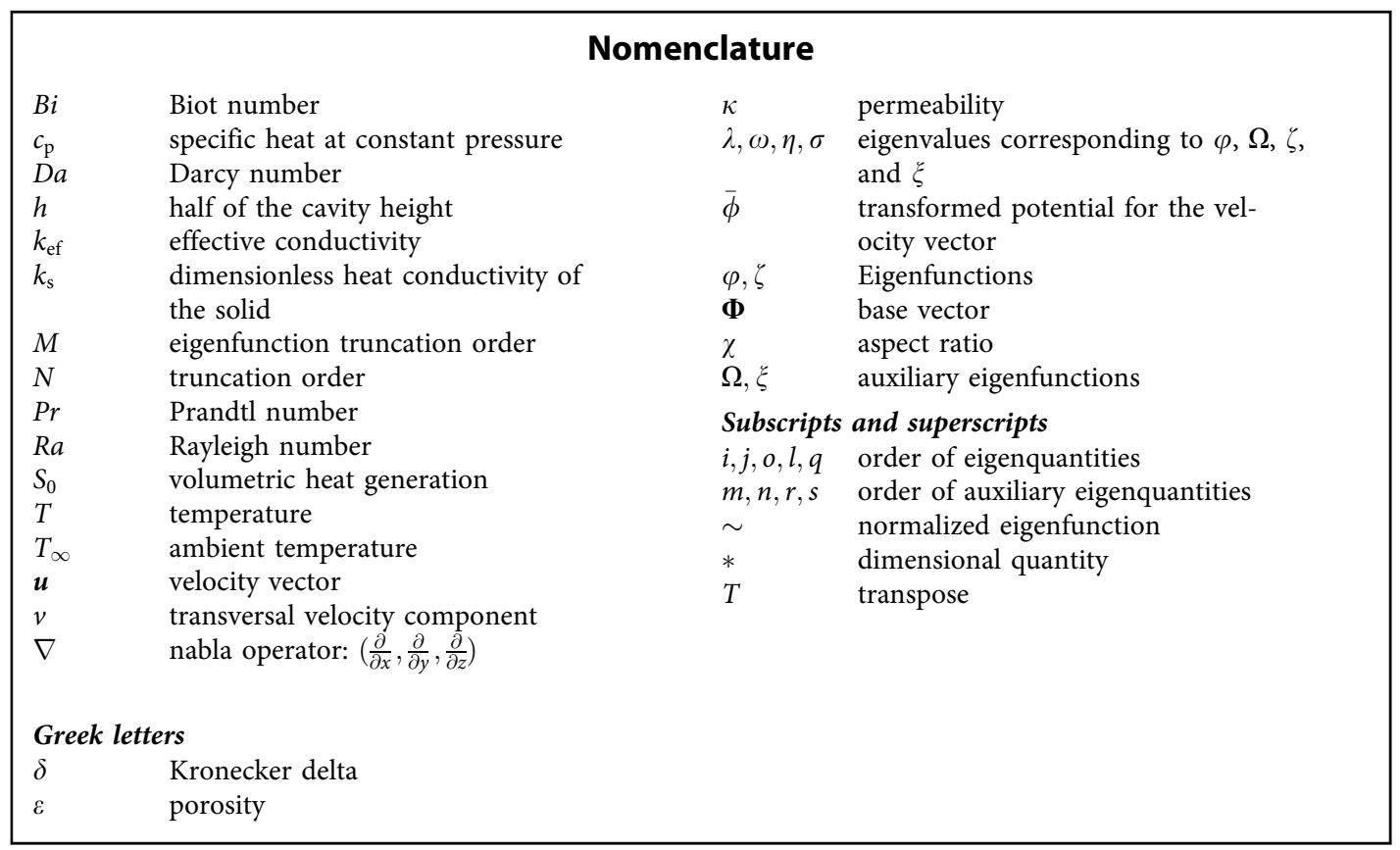

Despite the ubiquitous presence of purely numerical schemes for the solution of partial differential equations, analytical solutions are still needed for benchmarking and low-cost, arbitrarily precise calculations. Nevertheless, analytical methods are usually restricted to linear problems in regular geometries, which severely limits their applicability. In this context, the so-called hybrid numerical-analytical methods were proposed, as a means to enable the extension of analytical techniques to applications once dominated by finite elements and finite volumes analyses. Among the hybrid techniques devised over the years, the Generalized Integral Transform Technique (GITT) has proven to be a fairly general approach, enabling the solution of problems with moving boundaries, in irregular domains, with nonlinear boundary conditions and source terms, etc. By analytically treating all but one independent variable, the GITT enables automatic error control of the solution of partial differential equations. More complete descriptions of the theory and of the applications of the GITT can be found in several reference works [11-16]. The GITT has proven to be suitable to solve natural convection models in fluid filled cavities [17-19], adjacent fluid and porous layers [20, 21], and in cavities completely filled with porous media [22-24]. Other applications involving the integral transform solution of the Navier-Stokes equations in both primitive variables [25] and streamfunction-only [26-30] formulations are also readily available.

Recent developments in the GITT extended its applicability to complex configurations, once solvable essentially by purely numerical approaches. The so-called single domain formulation strategy allows for a more straightforward treatment of models involving heterogeneous media and complex geometries by the introduction of abruptly spatially varying physical properties and source terms [31-37]. Then, the associated eigenvalue problem carries the information on the space variable coefficients, which account for the transition at the various interfaces between different materials or geometric regions. This approach has been recently extended to the solution of the Navier-Stokes equations in heterogeneous media, with a successful application to both laminar internal flow and forced convection in a duct partially filled with porous media $[38,39]$. 
The present application was motivated by the wet storage of spent nuclear fuel elements and the passive cooling of the storage pool. The events leading to the Fukushima Daiichi accident have urged the nuclear engineering community to develop passive alternatives to remove the decay heat from nuclear fuel elements [40]. Among the facilities affected by the regulation that followed, the spent fuel pool (SFP) is here highlighted. In the absence of electrical power, the constant supply of fresh water from the active cooling system of the SFP stops, increasing the likelihood of reaction between the cladding of the fuel elements and water due to elevated temperatures $[41,42]$. Such an event could potentially expose the radioactive material with harmful consequences. To overcome these difficulties, heat pipes have been proposed as an effective passive heat removal system $[43,44]$. In fact, a heat pipes system for SFP is already in operation in a Nuclear Power Plant at Gösgen, Switzerland [45]. However, basic understanding of the effects of the heat pipes in the natural convection within SFPs is still lacking.

The direct numerical analysis of the nuclear fuel elements in SFPs can be quite cumbersome. To avoid such complications, a porous medium model is usually applied allowing for precise and computationally effective solutions to be obtained for intricate problems involving nuclear fuel elements. In this context, advances on the understanding of the effects of a passive cooling system on a SFP are here pursued. The GITT with single domain formulation is employed in the solution of the partial differential equations involved, whilst a finite element analysis (FEA) is carried out for co-verification purposes. A possible approach for this problem consists of the establishment of two different models for each layer, coupled by their common boundary. However, a more straightforward approach has been recently demonstrated for flow and mass transfer between porous and fluid layers $[38,39]$. Instead of treating each region separately, the single domain formulation mentioned above proposes a unique set of equations with abruptly varying physical properties to represent the transition between the different media. Besides the gain in computational time, the analytical pretreatment of the problem is greatly simplified, which renders the single domain formulation strategy particularly symbiotic with the GITT approach. The effects of the dimensionless temperature of the phase change refrigerant, of the Rayleigh number, and of the aspect ratio of the SFP are here investigated. Physical insights are drawn and some design directives are proposed, aiming at reduced maximum temperature within the cavity.

\section{Formulation and solution methodology}

\subsection{Fluid flow model}

Figure 1 depicts the physical situation under consideration for the fluid flow model. A rectangular cavity is partially filled with a water saturated porous medium representing the nuclear fuel elements, in accordance with the approach employed in previous works [41-43]. The remainder of the cavity is occupied by a layer of fluid. In the present illustration of the methodology, the porous and fluid layers fill half of the cavity each. A Cartesian coordinate system is included at the midpoint of the left vertical side of the cavity to facilitate the understanding of the model. Furthermore, some dimensions of interest are presented in Figure 1.

The fluid layer is assumed as an incompressible, steady-state, laminar flow of a Newtonian fluid with constant physical properties. The continuity and Navier-Stokes equations are deemed the appropriate model for this region. In addition, a buoyancy term with the Boussinesq approximation is included. In other words, the fluid density is considered to be constant except in the body forces term, in which a linearized compressibility relation is used. As for the porous layer, a Darcy model with a first order Brinkman correction is employed [6,7]. The presence of the Brinkman correction allows the imposition of continuity boundary conditions for the velocity, normal stress, and tangential stress vectors at the 


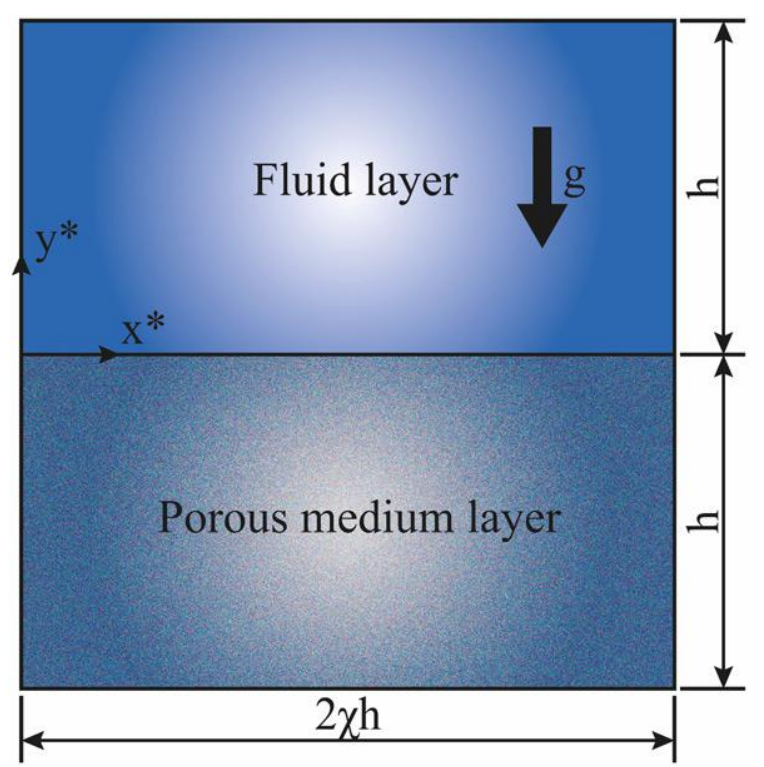

Figure 1. Schematic representation of the rectangular cavity partially filled with a porous medium generating heat and representing the spent nuclear fuel elements.

interface between the fluid and porous layers $[8,9,20,21]$. Thus, the fluid flow model can be written in dimensionless form as:

$$
\begin{gathered}
\nabla \cdot \boldsymbol{u}=0 \\
\rho(\boldsymbol{u} \cdot \nabla) \boldsymbol{u}=-\nabla p+\nabla \cdot[\mu \nabla \boldsymbol{u}]-\frac{\gamma}{D a} \boldsymbol{u}+\frac{R a}{8 P r} T \boldsymbol{e}_{y}
\end{gathered}
$$

where $\boldsymbol{u}$ is the dimensionless velocity vector, $\boldsymbol{e}_{y}$ is the unitary vector in the $y$-direction, $\nabla$ is the dimensionless nabla operator, $\rho$ is the dimensionless density, $p$ is the dimensionless pressure, $\mu$ is the dimensionless dynamic viscosity, $T$ is the dimensionless temperature, $R a$ is the Rayleigh number based on the height of the cavity, $\operatorname{Pr}$ is the Prandtl number, and $D a$ is the Darcy number. The dimensionless quantities are obtained from their dimensional counterparts in the following way:

$$
\begin{gathered}
\boldsymbol{u}=\rho_{0} h \frac{\boldsymbol{u}^{*}}{\mu_{0}} ; \nabla=h \nabla^{*} ; \rho=\frac{\rho^{*}}{\rho_{0}} ; p=\rho_{0} h^{2} \frac{p^{*}}{\mu_{0}^{2}} ; T=\mu_{0} c_{\mathrm{p}} \frac{T^{*}-T_{\infty}}{h^{2} S_{0}} \\
\mu=\frac{\mu^{*}}{\mu_{0}} ; R a=\frac{8 \rho_{0} g \beta S_{0} h^{5}}{\mu_{0}{ }^{2} c_{\mathrm{p}} \alpha_{0}} ; \operatorname{Pr}=\frac{\mu_{0}}{\rho_{0} \alpha_{0}} ; D a=\frac{\kappa}{h^{2}}
\end{gathered}
$$

where $\boldsymbol{u}^{*}$ is the velocity vector, $\nabla^{*}$ is the nabla operator, $\rho^{*}$ is the density, $\rho_{0}$ is the fluid density, $p^{*}$ is the pressure, $T$ is the temperature, $T_{\infty}$ is a reference temperature, $\mu^{*}$ is the viscosity, $\mu_{0}$ is the fluid viscosity, $c_{\mathrm{p}}$ is the specific heat, $\alpha_{0}$ is the thermal diffusivity, $\beta$ is the volumetric expansion coefficient, $S_{0}$ is a characteristic volumetric heat generation rate of the nuclear fuel elements, $\kappa$ is the permeability of the porous medium, and $g$ is the gravitational acceleration.

In accordance with the single domain formulation, the physical properties of Eq. (2) vary abruptly across the interfaces between different media. Let $V_{\mathrm{f}}$ and $V_{\mathrm{p}}$ be the regions occupied by the fluid and porous layers, respectively. Employing the effective viscosity [7-9], neglecting the inertial terms within the porous medium, and disregarding the dissipative term in the fluid layer, the following definitions are adopted: 


$$
\begin{aligned}
& \rho= \begin{cases}1, & \text { in } V_{\mathrm{f}} \\
0, & \text { in } V_{\mathrm{p}}\end{cases} \\
& \mu= \begin{cases}1, \text { in } V_{\mathrm{f}} \\
1 / \varepsilon, \text { in } V_{\mathrm{p}}\end{cases} \\
& \gamma= \begin{cases}0, & \text { in } V_{\mathrm{f}} \\
1, & \text { in } V_{\mathrm{p}}\end{cases}
\end{aligned}
$$

where $\varepsilon$ is the porosity of the porous medium.

To complete the modeling for the fluid flow, a set of boundary conditions must be established. The Brinkman viscous correction allows for the imposition of no-slip boundary conditions at the four walls surrounding both the fluid and porous layers. At the interface between the two layers, the single domain formulation automatically imposes continuity of the velocity vector and of the normal and tangential stresses. Mathematically,

$$
\begin{gathered}
\boldsymbol{u}(0, y)=0 ; \boldsymbol{u}(2 \chi, y)=0 \\
\boldsymbol{u}(x,-1)=0 ; \boldsymbol{u}(x, 1)=0
\end{gathered}
$$

\subsection{Heat transfer model}

The energy equation with constant thermophysical properties and constant heat generation rate, only within the fuel elements region, is used to model the heat transfer phenomena. The single domain formulation is once more employed, thereby simplifying the set of equations through the introduction of abrupt variations of thermophysical properties and source term at the interface between the fluid layer and the fluid saturated porous medium. The resulting dimensionless model is shown below:

$$
\boldsymbol{u} \cdot \nabla T=\frac{1}{\operatorname{Pr}} \nabla \cdot[k \nabla T]+\gamma
$$

where $k$ is the dimensionless thermal conductivity, which can be obtained from its dimensional counterpart as follows:

$$
k=\frac{k^{*}}{k_{0}}
$$

where $k^{*}$ is the dimensional thermal conductivity and $k_{0}$ is the thermal conductivity of the fluid.

Following the single domain formalism, the dimensionless thermal conductivity is set to vary abruptly at the interface between the fluid layer and the porous layer. Mathematically,

$$
k=\left\{\begin{array}{c}
1, \text { in } V_{\mathrm{f}} \\
k_{\mathrm{ef}}, \text { in } V_{\mathrm{p}}
\end{array}\right.
$$

where $k_{\mathrm{ef}}$ is the dimensionless effective thermal conductivity of the fluid saturated porous medium. The effective thermal conductivity is then obtained by taking the volumetric mean value between the thermal conductivities of the fluid and solid phases, resulting in the following expression:

$$
k_{\mathrm{ef}}=\varepsilon+(1-\varepsilon) k_{\mathrm{s}}
$$

where $k_{\mathrm{s}}$ is the ratio between the thermal conductivity of the solid phase and that of the fluid phase.

To complete the heat transfer model, a set of boundary conditions must be established. The bottom side of the cavity is considered to be thermally insulated. The top side of the cavity is considered to be exposed to natural convection with the air layer just above it. The sidewalls are considered to exchange heat with heat exchangers. The temperature is assumed to be constant, in 
accordance with the phase change of the refrigerant employed in the heat pipes system [43]. In mathematical form, the boundary conditions become:

$$
\begin{gathered}
T(0, y)=T_{\mathrm{w}} ; T(2 \chi, y)=T_{\mathrm{w}} \\
\left.\frac{\partial T}{\partial y}\right|_{y=-1} ^{=0 ;} \operatorname{BiT}(x, 1)+\left.\frac{\partial T}{\partial y}\right|_{y=1}=0
\end{gathered}
$$

where $T_{\mathrm{w}}$ is the dimensionless temperature of phase change of the refrigerant and $B i$ is the Biot number based on the half height of the cavity, $h$.

\subsection{Eigenvalue problem for the fluid flow model}

Considering an infinite number of elemental vortices composing the velocity vector, as previously proposed [38], the velocity vector can be represented by the following expansion:

$$
\boldsymbol{u}(x, y)=\sum_{i=1}^{\infty} \nabla \times\left[\bar{\phi}_{i}(x) \boldsymbol{\Phi}_{i}(y)\right]
$$

Equation (13) automatically satisfies the continuity equation, Eq. (1), sharing the same advantage of the streamfunction-only formulation [26-30], though not restricted to two-dimensional problems.

To warrant convergence to an arbitrary precision, the orthogonality property is demanded from vector $\boldsymbol{\Phi}_{i}[11,38]$. Furthermore, the base vector $\boldsymbol{\Phi}_{i}$ should contain as much information from the physical situation as possible, in order to achieve fast convergence rates and to minimize the computational effort. The chosen eigenvalue problem with the aforementioned characteristics has already been reported [38], and the involved set of equations is reproduced below:

$$
\begin{gathered}
\frac{d^{2}}{d y^{2}}\left(\mu \frac{d^{2} \varphi_{i}}{d y^{2}}\right)-\frac{d}{d y}\left(\frac{\gamma}{D a} \frac{d \varphi_{i}}{d y}\right)=\lambda_{i}{ }^{4} \mu(y) \varphi_{i}(y) \\
\varphi_{i}(-1)=0 ;\left.\frac{d \varphi_{i}}{d y}\right|_{y=-1}=0 \\
\varphi_{i}(1)=0 ;\left.\frac{d \varphi_{i}}{d y}\right|_{y=1}=0 \\
\widetilde{\varphi}_{i}(y)=\frac{\varphi_{i}(y)}{\sqrt{N_{\varphi, i}}} ; N_{\varphi, i}=\int_{-1}^{1} \mu(y) \varphi_{i}(y)^{2} d y
\end{gathered}
$$

with the base vector $\boldsymbol{\Phi}_{i}$ defined as follows:

$$
\boldsymbol{\Phi}_{i(}(y)=\left[\begin{array}{lll}
0 & 0 & \widetilde{\varphi}_{i}(y)
\end{array}\right]^{T}
$$

The base vector bears the following orthogonality property [38]:

$$
\int_{-1}^{1} \mu(y) \boldsymbol{\Phi}_{i}(y) \cdot \boldsymbol{\Phi}_{j}(y) d y=\delta_{i j}
$$

In light of the difficulty in analytically solving the eigenvalue problem of Eqs. (14a-g) in terms of elementary functions, the GITT itself is employed as a hybrid solution methodology. This strategy has already been successfully applied in previous works, including those that adopt the single domain formulation [31-37]. To proceed with the GITT formalism in the solution of Eqs. $(14 \mathrm{a}-\mathrm{g})$, a simpler eigenvalue problem with known analytical solution is proposed. In this work, the simple biharmonic eigenvalue problem shown below is used [38]: 


$$
\begin{gathered}
\frac{d^{4} \Omega_{m}}{d y^{4}}=\omega_{\mathrm{i}}^{4} \Omega_{m}(y) \\
\Omega_{m}(-1)=0 ;\left.\frac{d \Omega_{m}}{d y}\right|_{y=-1}=0 \\
\Omega_{m}(1)=0 ;\left.\frac{d \Omega_{m}}{d y}\right|_{y=1}=0
\end{gathered}
$$

with normalized eigenfunction and normalization integral given, respectively, by

$$
\widetilde{\Omega}_{m}(y)=\frac{\Omega_{m}(y)}{\sqrt{N_{\Omega, m}}} ; N_{\Omega, m}=\int_{-1}^{1} \Omega_{m}(y)^{2} d y
$$

The simpler eigenvalue problem of Eqs. $(17 \mathrm{a}-\mathrm{g})$ admits the following solution:

$$
\widetilde{\Omega}_{m}(y)=\frac{1}{\sqrt{2}}\left\{\begin{array}{l}
\frac{\cos \left(\omega_{m} y\right)}{\cos \left(\omega_{m}\right)}-\frac{\cosh \left(\omega_{m} y\right)}{\cosh \left(\omega_{m}\right)} \text { if } m \text { odd } \\
\frac{\sin \left(\omega_{m} y\right)}{\sin \left(\omega_{m}\right)}-\frac{\sinh \left(\omega_{m} y\right)}{\sinh \left(\omega_{m}\right)} \text { if } m \text { even }
\end{array}\right.
$$

where $\omega_{m}$ 's are solution to the transcendental equation below:

$$
\tanh \left(\omega_{m}\right)=\left\{\begin{array}{c}
-\tan \left(\omega_{m}\right) \text { if } m \text { odd } \\
\tan \left(\omega_{m}\right) \text { if } m \text { even }
\end{array}\right.
$$

bearing the orthogonality property below:

$$
\int_{-1}^{1} \widetilde{\Omega}_{m}(y) \widetilde{\Omega}_{n}(y) d y=\delta_{m n}
$$

The orthogonality property of Eq. (18c) allows for the establishment of the following transform-inverse pair:

$$
\bar{\varphi}_{i, m}=\int_{-1}^{1} \widetilde{\Omega}_{m}(y) \varphi_{i}(y) d y ; \quad \varphi_{i}(y)=\sum_{m=1}^{\infty} \bar{\varphi}_{i, m} \widetilde{\Omega}_{m}(y)
$$

Proceeding with the integral transformation process, Eq. (14a) is operated on with $\int_{-1}^{1} \widetilde{\Omega}_{m}(y)_{-} d y$, resulting in the transformed algebraic eigenvalue problem below:

$$
\begin{gathered}
\left(P-\lambda_{i}^{2} Q\right) \bar{\varphi}_{i}=0 \\
\bar{\varphi}_{i}=\left\{\bar{\varphi}_{i, m}\right\} ; P=\left\{P_{m n}\right\} ; Q=\left\{Q_{m n}\right\} \\
P_{m n}=\int_{-1}^{1}\left[\mu \frac{d^{2} \widetilde{\Omega}_{m}}{d y^{2}} \frac{d^{2} \widetilde{\Omega}_{n}}{d y^{2}}+\frac{\gamma}{D a} \frac{d \widetilde{\Omega}_{m}}{d y} \frac{d \widetilde{\Omega}_{n}}{d y}\right] d y \\
Q_{m n}=\int_{-1}^{1} \mu \widetilde{\Omega}_{m} \widetilde{\Omega}_{n} d y
\end{gathered}
$$

After truncation to a finite order $M$, the algebraic eigenvalue problem of Eqs. (20a-f) can be solved with well-established routines available in numerical subroutine packages such as the Eigensystem function of the Mathematica v.10.4 platform. The resulting eigenvectors are then substituted into the inverse formula of Eq. (19b) to render the final expression for the eigenfunction $\varphi_{i}(y)$. 


\subsection{Eigenvalue problem for the heat transfer model}

Similarly, an eigenvalue problem must be proposed for the solution of the heat transfer model through integral transforms. Applying separation of variables to a simplified version of Eq. (8) without internal heat generation and convective terms, a Sturm-Liouville eigenvalue problem can be obtained for the $y$-direction eigenfunction expansion [11]. This eigenvalue problem enables the analytical solution of the elliptical partial differential equation that results and desirably contains all the information concerned with the abrupt variation of thermal conductivity at the interface between fluid and porous layers. The set of equations obtained through the process above described is shown below:

$$
\begin{aligned}
& \frac{d}{d y}\left[k(y) \frac{d \zeta_{l}}{d y}\right]+\eta_{l}^{2} k(y) \zeta_{l}(y)=0 \\
& \zeta_{l}^{\prime}(-1)=0 ; B i \zeta_{l}(1)+\zeta_{l}^{\prime}(1)=0
\end{aligned}
$$

with normalized eigenfuncitons and normalization integral given, respectively, by

$$
\widetilde{\zeta}_{l}(y)=\frac{\zeta_{l}(y)}{\sqrt{N_{\zeta, l}}} ; N_{\zeta, l}=\int_{-1}^{1} k(y) \zeta_{l}(y)^{2} d y
$$

The eigenfunction resulting from the solution of Eqs. (21a-e) has the following orthogonality property:

$$
\int_{-1}^{1} k(y) \widetilde{\zeta}_{l}(y) \widetilde{\zeta}_{q}(y) d y=\delta_{l q}
$$

The orthogonality property allows for the establishment of a transform-inverse pair for the dimensionless temperature as follows:

$$
\bar{T}_{l}(x)=\int_{-1}^{1} k(y) \widetilde{\zeta}_{l}(y) T(x, y) d y ; \quad T(x, y)=\sum_{l=1}^{\infty} \bar{T}_{l}(x) \widetilde{\zeta}_{l}(y)
$$

Again, the GITT approach is used for the solution of the eigenvalue problem for the heat transfer model. For this purpose, a simpler eigenvalue problem must be used in the expansion for $\widetilde{\zeta}_{l}$. For the heat transfer model, the simplest possible Sturm-Liouville problem is chosen, in the form:

$$
\begin{gathered}
\frac{d^{2} \xi_{r}}{d y^{2}}+\sigma_{r}^{2} \xi_{r}(y)=0 \\
\xi_{r}^{\prime}(-1)=0 ; B i \xi_{r}(1)+\xi_{r}^{\prime}(1)=0
\end{gathered}
$$

with normalized eigenfunctions and normalization integrals as

$$
\widetilde{\xi}_{r}=\frac{\xi_{r}(y)}{\sqrt{N_{\xi, r}}} ; N_{\xi, r}=\int_{-1}^{1} \xi_{r}(y)^{2} d y
$$

The following orthogonality property is valid for the solution of the eigenvalue problem of Eqs. (24a-e):

$$
\int_{-1}^{1} \widetilde{\xi}_{r}(y) \widetilde{\xi}_{s}(y) d y=\delta_{r s}
$$

A transform-inverse pair can then be formed for the eigenfunction $\zeta_{l}$,

$$
\bar{\zeta}_{l, r}=\int_{-1}^{1} \widetilde{\xi}_{r}(y) \zeta_{l}(y) d y ; \zeta_{l}(y)=\sum_{r=1}^{\infty} \bar{\zeta}_{l, r} \widetilde{\xi}_{r}(y)
$$


Operating Eq. (21a) with $\int_{-1}^{1} \widetilde{\xi}_{r}(y)_{-} d y$, using integration by parts to avoid derivatives of discontinuous functions, and employing the inverse formula of Eq. (26b), the algebraic eigenvalue problem below results:

$$
\begin{gathered}
\left(R+\eta_{l}^{2} U\right) \bar{\zeta}_{l}=0 \\
\bar{\zeta}_{i}=\left\{\bar{\zeta}_{i, r}\right\} ; \quad R=\left\{R_{r s}\right\} ; U=\left\{U_{r s}\right\} \\
R_{r s}=-\int_{-1}^{1} k(y) \frac{d \widetilde{\xi}_{r}}{d y} \frac{d \widetilde{\xi}_{s}}{d y} d y \\
U_{r s}=\int_{-1}^{1} k(y) \widetilde{\xi}_{r} \widetilde{\xi}_{s} d y
\end{gathered}
$$

Upon truncation to a finite order $\hat{M}$, the algebraic eigenvalue problem of Eqs. $(27 \mathrm{a}-\mathrm{f})$ is solved with the routine Eigensystem built-in the Mathematica v.10.4 platform. Additionally, the software Mathematica is used to obtain the numerical values of the coefficients of Eqs. $(27 \mathrm{e}, \mathrm{f})$ through analytically determined expressions. The eigenvectors are then substituted into the inverse formula Eq. (26b) to fully determine the eigenfunctions $\zeta_{l}$.

\subsection{Transformed problem}

The eigenfunction expansion proposed in Eq. (13) identically satisfies the continuity equation, Eq. (1). Therefore, the only remaining equations are the ones related to linear momentum and energy conservations. Proceeding then with the integral transforms formalism, Eq. (2) is operated with $\int_{d V}\left(\nabla \times \boldsymbol{\Phi}_{i}\right) \cdot{ }_{-} d v$ leading to [38]:

$$
\int_{d V} \boldsymbol{\Phi}_{i} \cdot\left\{\nabla \times[\rho(\boldsymbol{u} \cdot \nabla) \boldsymbol{u}]-\nabla \times[\nabla \cdot(\mu \nabla \boldsymbol{u})]+\frac{1}{D a} \nabla \times[\gamma \boldsymbol{u}]-\frac{R a}{8 \operatorname{Pr}} \frac{\partial T}{\partial x} \boldsymbol{e}_{z}\right\} d v=0
$$

where $d V$ is an infinitesimal volume consisting of the whole vertical cross-section of the cavity depicted in Figure 1 and with an infinitesimal longitudinal length $d x$. The choice of the operator used in the integral transformation allows for the complete elimination of the pressure term, thus dropping the requirement to establish a separate eigenfunction expansion with the sole purpose of determining the pressure field [38].

Employing the inverse formulae of Eqs. (13) and (23b), using some tensor calculus identities, and rearranging, the following infinite system of coupled ordinary differential equations results [38]:

$$
\begin{gathered}
\frac{d^{4} \bar{\phi}_{i}}{d x^{4}}=-\lambda_{i}^{4} \bar{\phi}_{i}-\sum_{j=1}^{\infty} F_{i j} \frac{d^{2} \bar{\phi}_{j}}{d x^{2}}+\frac{R a}{8 \operatorname{Pr}} \sum_{l=1}^{\infty} G_{i l} \frac{d \bar{T}_{l}}{d x}- \\
-\sum_{o=1}^{\infty} \sum_{j=1}^{\infty}\left(A_{i j o} \frac{d \bar{\phi}_{j}}{d x} \bar{\phi}_{o}+B_{i j o} \bar{\phi}_{j} \frac{d \bar{\phi}_{o}}{d x}+C_{i j o} \frac{d^{2} \bar{\phi}_{j}}{d x^{2}} \frac{d \bar{\phi}_{o}}{d x}++D_{i j o} \frac{d \bar{\phi}_{j}}{d x} \frac{d^{2} \bar{\phi}_{o}}{d x^{2}}+E_{i j o} \frac{d^{3} \bar{\phi}_{j}}{d x^{3}} \bar{\phi}_{o}\right)
\end{gathered}
$$

with integral coefficients,

$$
\begin{gathered}
A_{i j o}=\int_{-1}^{1} \rho \frac{d \widetilde{\varphi}_{i}}{d y} \frac{d \widetilde{\varphi}_{j}}{d y} \frac{d \widetilde{\varphi}_{o}}{d y} d y \\
B_{i j o}=-\int_{-1}^{1} \rho \frac{d \widetilde{\varphi}_{i}}{d y} \frac{d^{2} \widetilde{\varphi}_{j}}{d y^{2}} \widetilde{\varphi}_{o} d y \\
C_{i j o}=\int_{-1}^{1}\left(\rho \widetilde{\varphi}_{i} \frac{d \widetilde{\varphi}_{j}}{d y} \widetilde{\varphi}_{o}-\rho \widetilde{\varphi}_{i} \widetilde{\varphi}_{j} \frac{d \widetilde{\varphi}_{o}}{d y}\right) d y
\end{gathered}
$$




$$
\begin{gathered}
D_{i j o}=\int_{-1}^{1} \rho \widetilde{\varphi}_{i} \frac{d \widetilde{\varphi}_{j}}{d y} \widetilde{\varphi}_{o} d y \\
E_{i j o}=-\int_{-1}^{1} \rho \widetilde{\varphi}_{i} \widetilde{\varphi}_{j} \frac{d \widetilde{\varphi}_{o}}{d y} d y \\
F_{i j}=-\int_{-1}^{1}\left[2 \mu \frac{d \widetilde{\varphi}_{i}}{d y} \frac{d \widetilde{\varphi}_{j}}{d y}+\frac{\gamma}{D a} \widetilde{\varphi}_{i} \widetilde{\varphi}_{j}\right] d y \\
G_{i l}=\int_{-1}^{1} \widetilde{\varphi}_{i} \widetilde{\zeta}_{l} d y
\end{gathered}
$$

A similar procedure is used for the integral transformation of the boundary conditions of Eqs. (7a,b) [38], yielding:

$$
\begin{gathered}
\bar{\phi}_{i}(0)=0 ; \quad \bar{\phi}_{i}^{\prime}(0)=0 \\
\bar{\phi}_{i}(2 \chi)=0 ; \quad \bar{\phi}_{i}^{\prime}(2 \chi)=0
\end{gathered}
$$

The integral transformation process of the energy conservation equation follows the usual formalism of the GITT. Applying $\int_{-1}^{1} \widetilde{\zeta}_{l}(y)_{-} d y$ to Eq. (8) expanded in the Cartesian coordinate system of Figure 1, using the inverse formulae of Eqs. (13) and (23b), and rearranging, we then have:

$$
\frac{d^{2} \bar{T}_{l}}{d x^{2}}=\eta_{l}^{2} \bar{T}_{l}+\operatorname{Pr} \sum_{o=1}^{\infty} \sum_{q=1}^{\infty}\left[A A_{l q o} \frac{d \bar{T}_{q}}{d x} \bar{\phi}_{o}+B B_{l q o} \bar{T}_{q} \frac{d \bar{\phi}_{o}}{d x}\right]-g_{l} \operatorname{Pr}
$$

with integral coefficients,

$$
\begin{aligned}
A A_{l q o} & =\int_{-1}^{1} \widetilde{\zeta}_{l} \widetilde{\zeta}_{q} \frac{d \widetilde{\varphi}_{o}}{d y} d y \\
B B_{l q o} & =-\int_{-1}^{1} \widetilde{\zeta}_{l} \frac{\widetilde{\zeta}_{q}}{d y} \widetilde{\varphi}_{o} d y \\
g_{l} & =\int_{-1}^{1} \widetilde{\zeta}_{l} \gamma d y
\end{aligned}
$$

Similarly, employing the operator $\int_{-1}^{1} k(y) \widetilde{\zeta}_{l}(y)_{-} d y$ into Eqs. (12a,b), the transformed boundary conditions are obtained as follows:

$$
\bar{T}_{l}(0)=\bar{T}_{l}(2 \chi)=T_{w} \int_{-1}^{1} k(y) \widetilde{\zeta}_{l}(y) d y
$$

\subsection{Solution procedure}

In face of the impossibility of solving two infinite sets of equations such as Eqs. (29a-h) and (31a-d), a truncation process must be carried out. The summations in the inverse formulae of Eqs. (13) and (23b) are limited to the orders $N$ and $\hat{N}$, respectively. The truncation is then propagated to the summations in Eqs. (29a) and (31a), limiting their order accordingly. The convergence of the results can be verified by monitoring these two truncation orders only [11].

The integrations in Eqs. (29b-h), (31b-d), and (32a,b) are all analytically determined, after substitution of the inverse formulae Eqs. (19b) and (26b), using symbolic computation functions available at the Mathematica v.10.4 platform. The resulting system of ordinary differential equations for the transformed potentials is then solved using the built-in function NDSolve of the Mathematica v.10.4 
Table 1. Convergence of the last five employed eigenvalues for the velocity vector and temperature eigenfunction expansions. $D a=10^{-5} ; \varepsilon=0.5 ; k_{\mathrm{s}}=8.33 ; B i=50$.

\begin{tabular}{lccccc}
\hline & $\lambda_{17}$ & $\lambda_{18}$ & $\lambda_{19}$ & $\lambda_{20}$ & $\lambda_{21}$ \\
\hline$M=183$ & 45.470 & 48.382 & 49.651 & 51.794 & 54.713 \\
$M=192$ & 45.468 & 48.381 & 49.650 & 51.792 & 54.711 \\
$M=195$ & 45.467 & 48.380 & 49.650 & 51.792 & 54.711 \\
$M=198$ & 45.467 & 48.380 & 49.650 & 51.791 & 54.710 \\
$M=201$ & 45.467 & 48.379 & 49.650 & 51.791 & 54.710 \\
\hline & $\eta_{17}$ & $\eta_{18}$ & $\eta_{19}$ & $\eta_{20}$ & $\eta_{21}$ \\
$\hat{M}=132$ & 25.385 & 27.572 & 28.511 & 30.686 & 31.639 \\
$\hat{M}=141$ & 25.384 & 27.570 & 28.511 & 30.683 & 31.638 \\
$\hat{M}=144$ & 25.384 & 27.569 & 28.510 & 30.682 & 31.638 \\
$\hat{M}=147$ & 25.384 & 27.568 & 28.510 & 30.682 & 31.638 \\
$\hat{M}=150$ & 25.384 & 27.568 & 28.510 & 30.681 & 31.638 \\
\hline
\end{tabular}

platform. However, for that purpose, a pseudo-transient term is introduced on the r.h.s. of Eqs. (29a) and (31a). The pseudo-transient term consists of the plain time-derivative of the transformed potential at the order $i$ and $l$ for Eqs. (29a) and (31a), respectively. Moreover, initial conditions consistent with the boundary conditions are imposed, i.e., zero for the transformed velocity vector and equal to the r.h.s. of Eq. (32a,b) for the transformed temperature. The pseudo-time interval is determined $a$ posteriori, aiming at achieving steady-state. The resulting transformed potentials at the end of the pseudo-time interval is then substituted into the inverse formulae of Eqs. (13) and (23b) to determine the velocity vector and temperature fields throughout the cavity.

\section{Results and discussion}

Along the present analysis, some parameters are kept constant throughout the test cases. The Darcy number and the porosity, for instance, were set to $10^{-5}$ and 0.5 , respectively, consistent with spent nuclear fuel elements assemblies, as estimated in the literature [43]. Similar arguments were used to determine a suitable value for $k_{s}$ as 8.33 . The Prandtl and Biot numbers are set to 7 (water) and 50, respectively.

First, the effects of the Rayleigh number $(\mathrm{Ra})$ are investigated and a thorough analysis under the laminar flow regime is performed, with five values of $\mathrm{Ra}$ ranging from 10 to $10^{5}$. Furthermore, a combined analysis of the effects of the Rayleigh number and of the dimensionless phase change temperature of the refrigerant is carried out. Three values of $T_{w}$ are used for that purpose: $0,0.5$, and 1 . Finally, the effect of the aspect ratio $(\chi)$ on the flow pattern and on the temperature distribution is investigated. Besides the aspect ratio of 2 used in the other analyses, the values of 4 and 6 are also employed for $\chi$. The same cases are analyzed with the software COMSOL Multiphysics v.5.2 (Burlington, MA) for co-verification.

\subsection{Convergence analysis}

Before physically analyzing the results obtained, the accuracy of the developed solution will be demonstrated through a convergence analysis. First, the convergence of the eigenvalue problems solution is investigated as a function of the truncation orders $M$ and $\hat{M}$. Table 1 shows the eigenvalues $\lambda_{i}$ and $\eta_{l}$ with increasing truncation orders, and a convergence to at least 5 significant digits can be inferred from the results for both sets of eigenvalues. Only the last 5 eigenvalues effectively used for the eigenfunction expansions of the velocity vector and the temperature are presented, on the assumption they show a slower convergence behavior in comparison with the lower order ones.

Regarding the convergence behavior of the velocity vector and temperature eigenfunction expansions, Table 2 shows the values for both transversal velocity component and temperature at selected positions within the cavity for Rayleigh numbers $10^{3}$ and $10^{5}$. Convergence to at least 
Table 2. Convergence of the transversal velocity component and of the temperature at four selected positions within the cavity for $R a=10^{5}, T_{\mathrm{w}}=0.5$, and $\chi=2$. Results presented for four $x$ positions along the line $y=0.5$.

\begin{tabular}{|c|c|c|c|c|c|c|c|c|}
\hline \multirow[t]{2}{*}{$\bar{N} / \hat{N}$} & \multicolumn{2}{|l|}{$x=0.5$} & \multicolumn{2}{|c|}{$x=1.7$} & \multicolumn{2}{|l|}{$x=2.3$} & \multicolumn{2}{|l|}{$x=3.5$} \\
\hline & $v$ & $T$ & $v$ & $T$ & $v$ & $T$ & $v$ & $T$ \\
\hline \multicolumn{9}{|c|}{$R a=10^{3}$} \\
\hline 3 & -0.027 & 0.678 & 0.023 & 1.149 & 0.023 & 1.149 & -0.027 & 0.678 \\
\hline 6 & -0.027 & 0.680 & 0.023 & 1.152 & 0.023 & 1.152 & -0.027 & 0.680 \\
\hline 9 & -0.027 & 0.682 & 0.023 & 1.153 & 0.023 & 1.153 & -0.027 & 0.682 \\
\hline 12 & -0.027 & 0.681 & 0.023 & 1.152 & 0.023 & 1.152 & -0.027 & 0.681 \\
\hline 15 & -0.027 & 0.681 & 0.023 & 1.152 & 0.023 & 1.152 & -0.027 & 0.681 \\
\hline 18 & -0.027 & 0.681 & 0.023 & 1.152 & 0.023 & 1.152 & -0.027 & 0.681 \\
\hline \multicolumn{9}{|c|}{$R a=10^{5}$} \\
\hline 3 & -1.907 & 0.53 & 2.032 & 0.994 & 2.033 & 0.994 & -1.908 & 0.53 \\
\hline 6 & -1.907 & 0.553 & 2.018 & 0.925 & 2.019 & 0.925 & -1.907 & 0.553 \\
\hline 9 & -1.908 & 0.571 & 2.023 & 0.909 & 2.024 & 0.909 & -1.908 & 0.571 \\
\hline 12 & -1.907 & 0.562 & 2.021 & 0.913 & 2.022 & 0.913 & -1.907 & 0.562 \\
\hline 15 & -1.907 & 0.563 & 2.022 & 0.918 & 2.023 & 0.918 & -1.907 & 0.563 \\
\hline 18 & -1.907 & 0.563 & 2.022 & 0.916 & 2.022 & 0.916 & -1.907 & 0.563 \\
\hline 21 & -1.907 & 0.563 & 2.022 & 0.915 & 2.022 & 0.915 & -1.907 & 0.563 \\
\hline
\end{tabular}

three significant digits is achieved for the condition shown, with truncation orders $N$ and $\hat{N}$ at most equal to 21 . These values shall be used to generate all forthcoming results.

\subsection{Natural convection for different Rayleigh numbers and refrigerant phase change temperatures}

Figure $2 \mathrm{a}-\mathrm{c}$ depicts the streamlines within the cavity for different Rayleigh numbers with a dimensionless phase change temperature of the refrigerant of 0.5 . Two symmetrical natural convection cells can be observed in the fluid layer for the three values of $R a$ considered, whilst in the porous layer the fluid movement is comparatively weak. This behavior was expected due to the magnitude of the Darcy number, preventing significant flow to develop below the line $y=0$. The streamlines for both natural convection cells for $R a=10^{4}$ get closer at the middle of the cavity and develop steeper gradients in comparison with the $R a=10^{3}$ case. For $R a=10^{5}$, a horizontal stretching of the cells develops, together with further enhancement of the gradients, indicating a much larger magnitude of the velocity vectors. The temperature contours of Figure $2 \mathrm{~d}-\mathrm{f}$ corroborate the previous findings. Within the porous layer for $R a=10^{3}$, the vertically stratified pattern indicates a dominance of heat transfer by conduction. Furthermore, Figure $2 \mathrm{~d}-\mathrm{f}$ shows a progressive disruption of the elliptical behavior of the temperature contours within the fluid layer predominant on the cases with lower $R a$, consistent with a more relevant role of advection, as discussed in the analysis of the streamlines.

Figure $3 \mathrm{a}-\mathrm{f}$ show the transversal velocity and temperature profiles for three horizontal lines within the fluid layer and three different values of the Rayleigh number. A comparison with results obtained with the commercial software COMSOL Multiphysics v.5.2 (Burlington, MA) is offered, and a good agreement with the results from the integral transforms solution is evident in all cases considered. A downward movement near the sidewalls appears in the three cases of Rayleigh number used to generate the results of Figure $3 \mathrm{a}-\mathrm{c}$. This behavior is consistent with the formation of a natural convection boundary layer over a vertical cold wall. Moreover, steeper velocity gradients near the wall and towards the center of the cavity are confirmed, in accordance with previously stated conclusions.

Figure $4 \mathrm{a}$ shows the dependence of the maximum temperature within the pool with the Rayleigh number for three different values of the dimensionless phase change temperature of the refrigerant. As expected, lowering the temperatures imposed at the sidewalls renders the cooling 

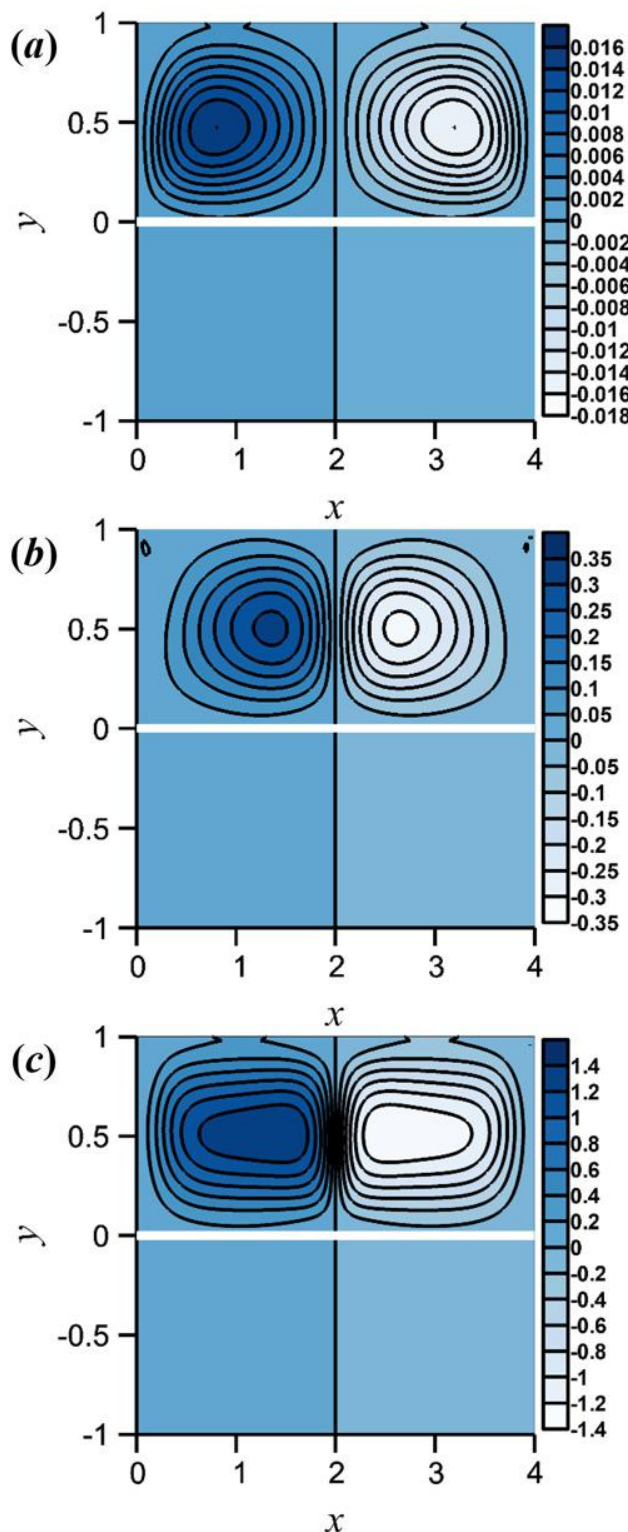
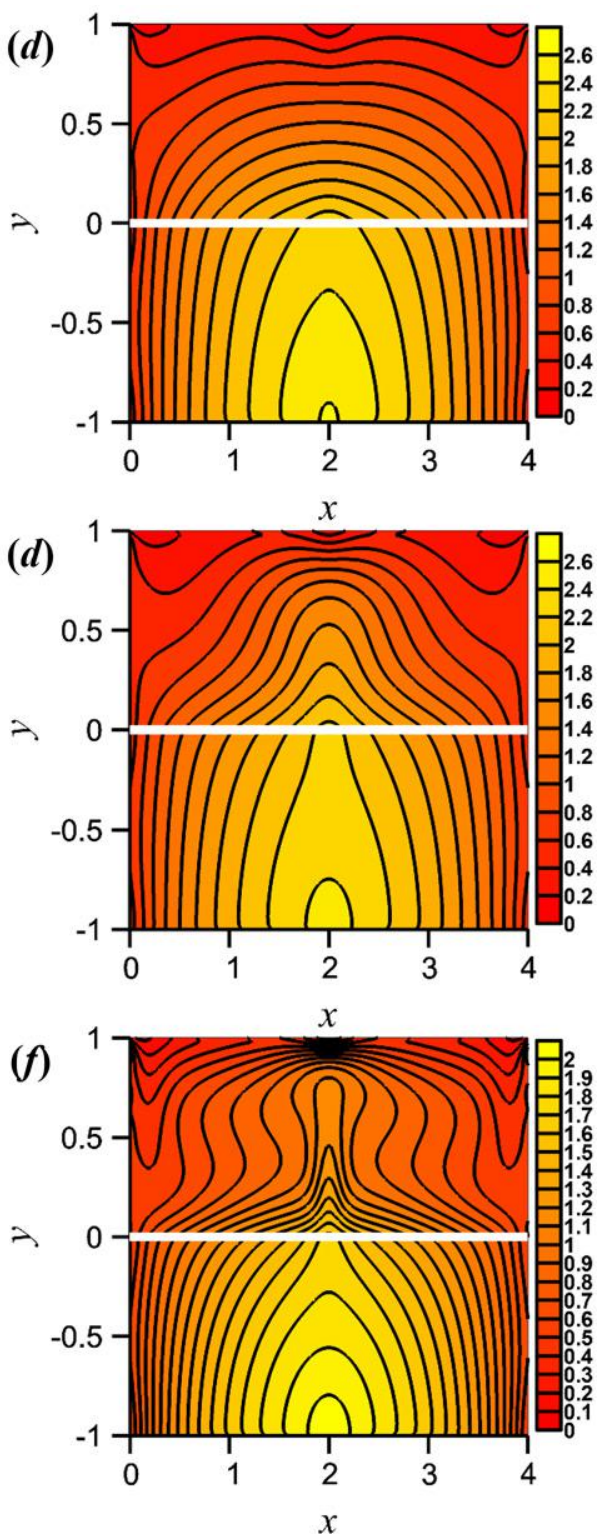

Figure 2. Streamlines and temperature contours for varying $R a$ and $T_{\mathrm{w}}=0.5$. Streamlines: (a) $\mathrm{Ra}=10^{3}$; (b) $\mathrm{Ra}=10^{4} ;$ (c) $\mathrm{Ra}=10^{5}$. Temperature: (d) $\mathrm{Ra}=10^{3}$; (e) $\mathrm{Ra}=10^{4}$; (f) $\mathrm{Ra}=10^{5}$. Horizontal lines identify the fluid-porous layers interface.

of the pool more efficient. The results of Figure $4 \mathrm{a}$ also show a nearly constant maximum temperature of the pool for $R a \leq 10^{3}$, indicating the heat transfer is dominated by conduction in this range, in accordance with findings stemming from Figure $2 \mathrm{a}-\mathrm{f}$. A drop of roughly $20 \%$ on the maximum temperature can be observed for higher Rayleigh numbers, indicating a more prominent role of advection on the heat transfer process. This decrease on the maximum temperature with rising Rayleigh numbers, allows for the confirmation that increments on $R a$, while maintaining the internal heat generation rate on the nuclear fuel elements constant, increases the cooling efficiency of the SFP. For instance, higher $R a$ can be easily achieved through deeper SFPs as suggested by Eq. (3g). Figure $4 \mathrm{~b}$ is aimed at further consolidating the understanding of transition 
(a)

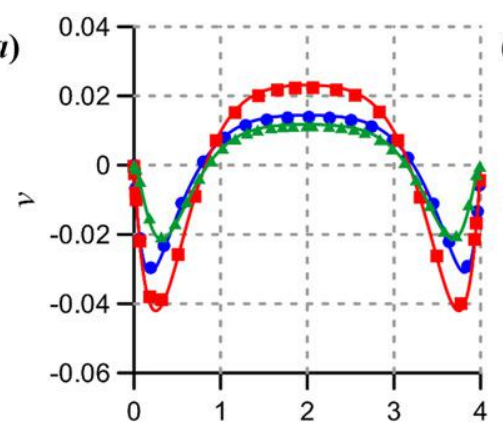

(b)

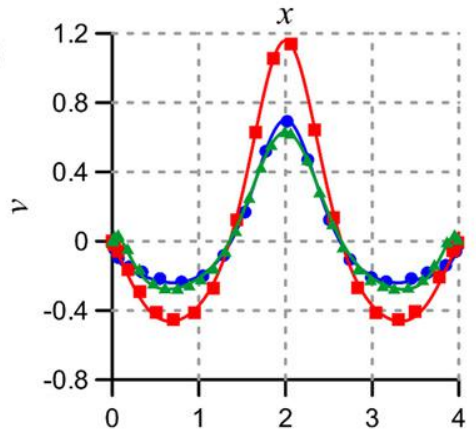

(c)

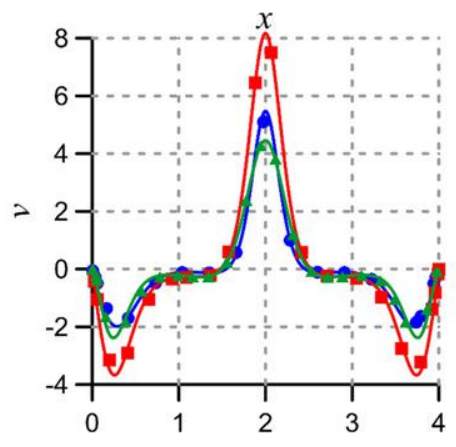

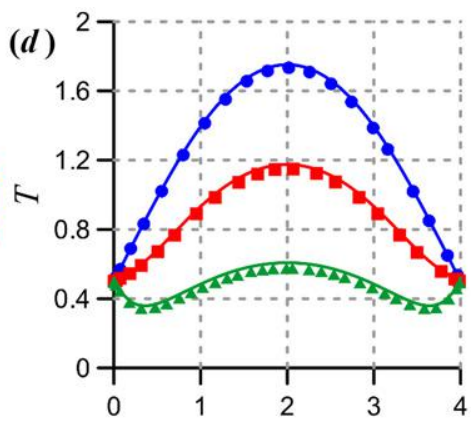

(e)

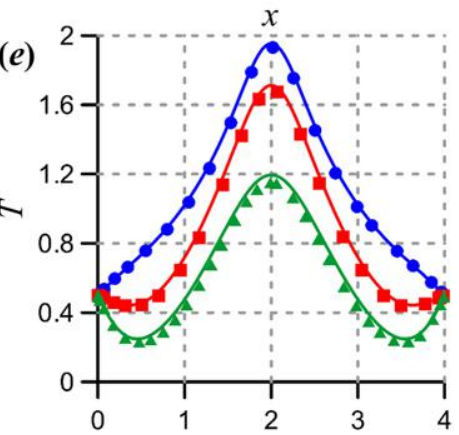

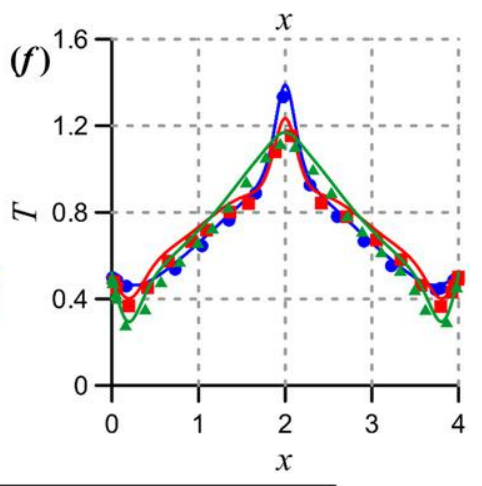

$\bullet \bullet \bullet y=0.25 \square \square \square y=0.5 \Delta \Delta \Delta y=0.75$

Figure 3. Transversal velocity component and temperature profiles for varying $R a$ and $T_{\mathrm{w}}=0.5$. (a) $\mathrm{Ra}=10^{3}$; (b) $\mathrm{Ra}=10^{4}$; (c) $\mathrm{Ra}$ $=10^{5}$; (d) $\mathrm{Ra}=10^{3}$; (e) $\mathrm{Ra}=10^{4}$; (f) $\mathrm{Ra}=10^{5}$. Symbols represent values obtained with COMSOL Multiphysics (Burlington, MA).

between a conduction dominated to a convection regime within the SFP. For that purpose, the maximum absolute value of the streamfunction is used as defined below:

$$
\psi_{\max }=\max _{x, y}\left|\sum_{i=1}^{\infty} \bar{\phi}_{i}(x) \boldsymbol{\Phi}_{i}(y)\right|
$$

The steep increase on the value of $\psi_{\max }$ for Rayleigh numbers higher than $10^{3}$ indicates the development of much stronger vortices, in accordance with the conclusion that this value is a reasonable approximation to the threshold where natural convection occurs in a more effective way. Nevertheless, only a mild variation of $\psi_{\max }$ with the sidewall temperature is observed in Figure $4 \mathrm{~b}$, indicating that, at least for the range of parameters here analyzed, the phase change temperature of the refrigerant has little effect on the strength of the natural convection cells. 


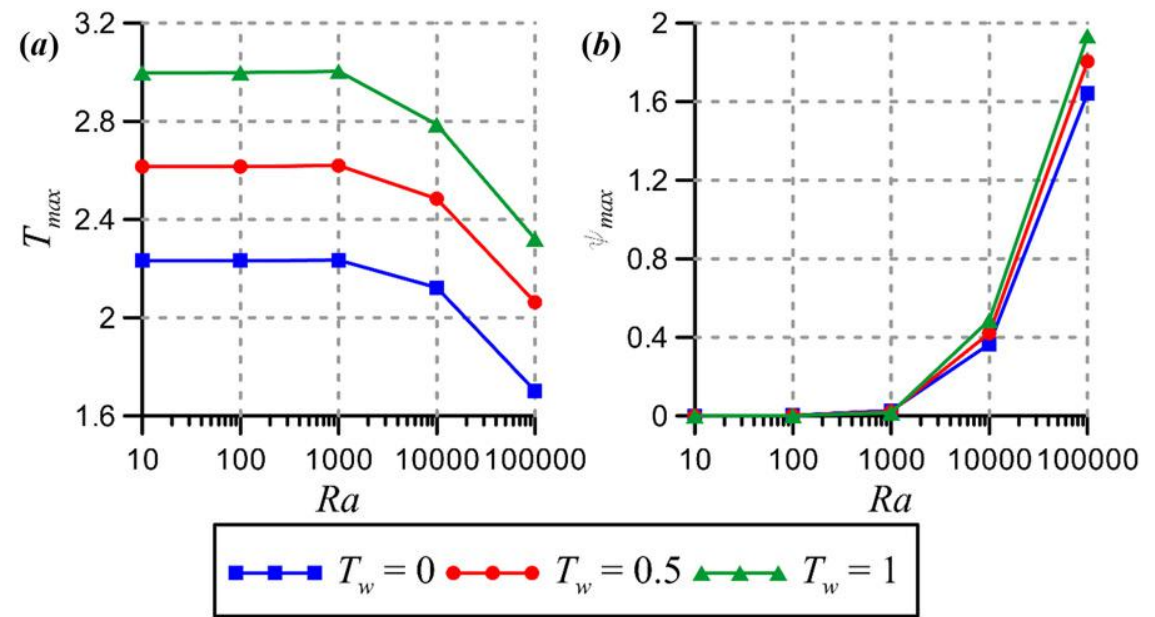

Figure 4. Maximum temperature and absolute streamfunction value for varying $R a$ and $T_{\mathrm{w}}$. (a) Maximum temperature; (b) Maximum absolute streamfunction value.
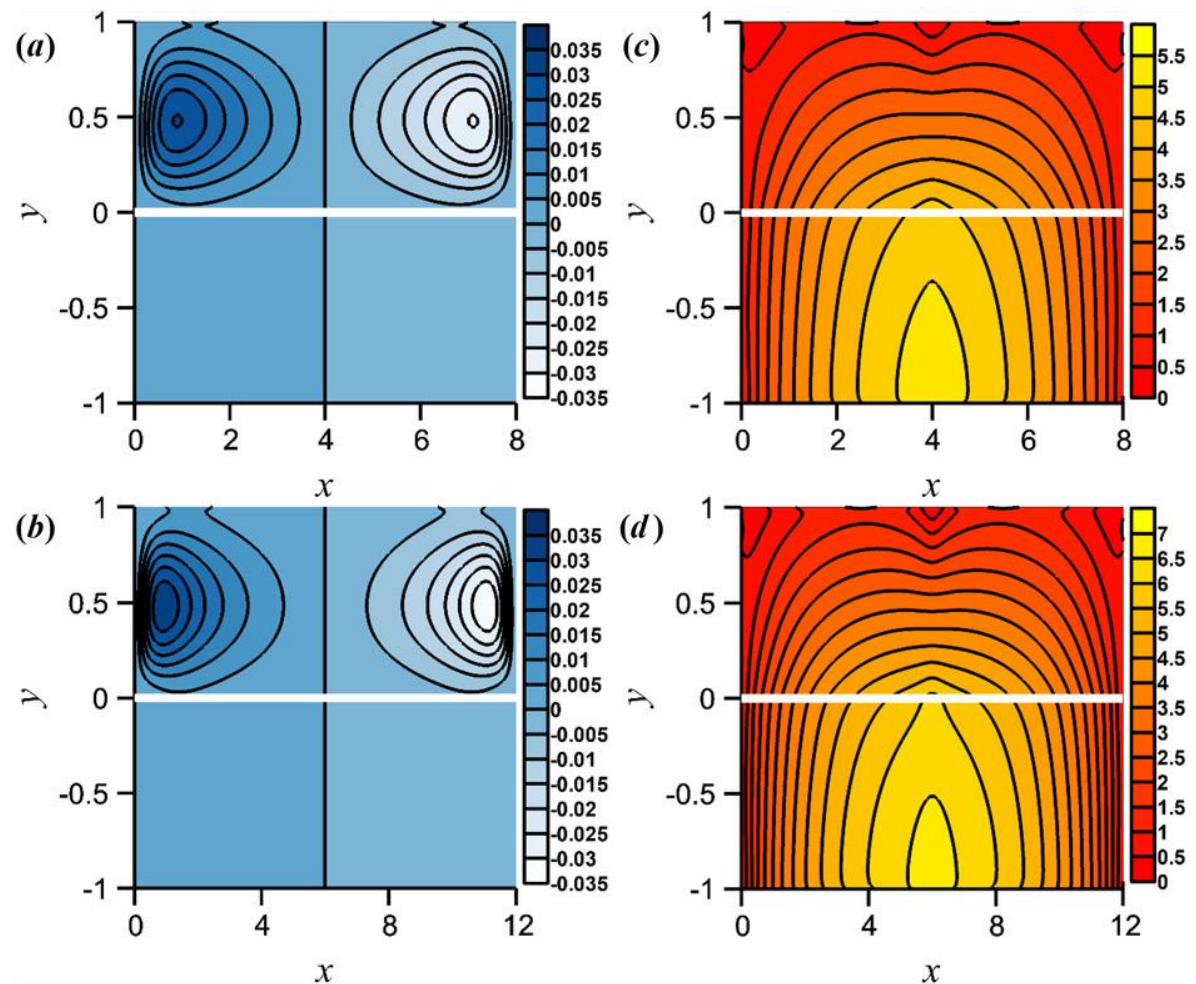

Figure 5. Streamlines and temperature contours for varying $\chi, R a=10^{3}$, and $T_{\mathrm{w}}=0.5$. Streamlines: (a) $\chi=4$; (b) $\chi=6$. Temperature: (c) $\chi=4 ;$ (d) $\chi=6$. Horizontal lines represent the fluid-porous layers interface.

\subsection{Natural convection for different aspect ratios}

Streamlines and temperature contours for varying aspect ratios are offered in Figure $5 \mathrm{a}-\mathrm{d}$, with $R a=10^{3}$ and $T_{\mathrm{w}}=0.5$. The pattern of the streamlines presented in Figures $2 \mathrm{a}$ and $5 \mathrm{a}, \mathrm{b}$ clearly shows the tendency of stronger vortices near the wall and an increasing area of uniform flow near the vertical centerline. On the other hand, Figures $2 \mathrm{~d}$ and $5 \mathrm{c}, \mathrm{d}$ are qualitatively 
(a)

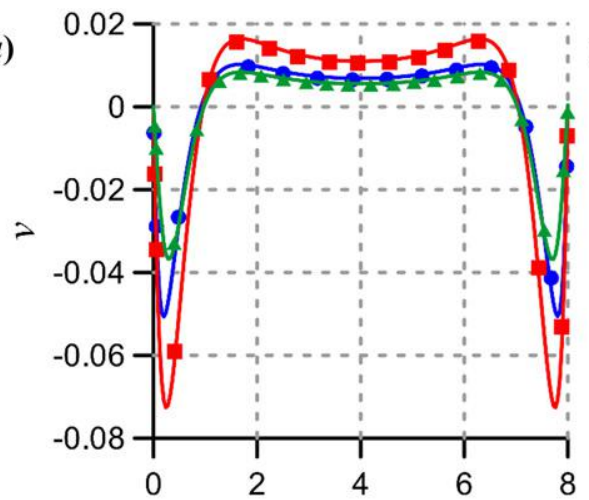

(b)

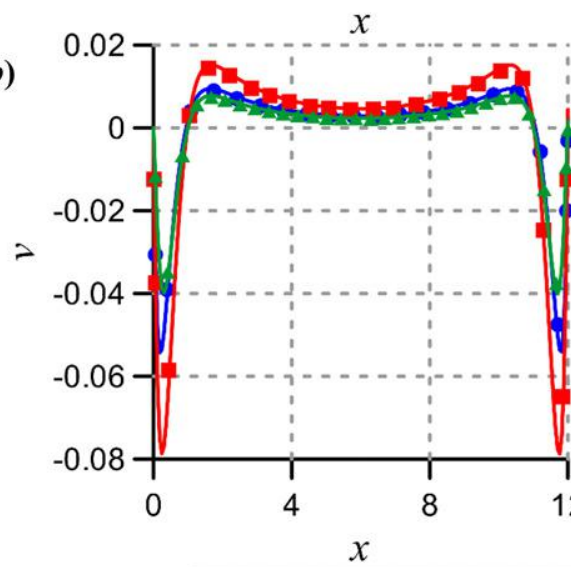

(c)

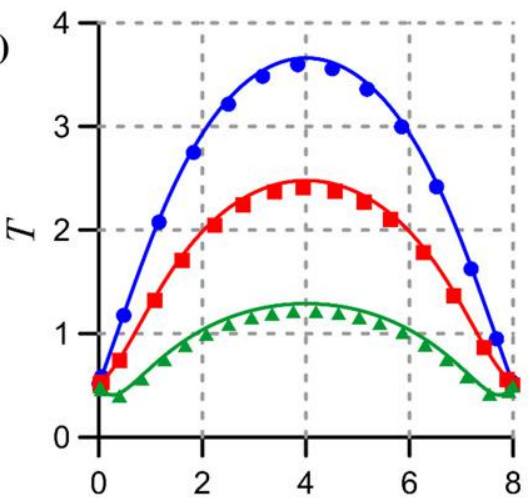

(d)

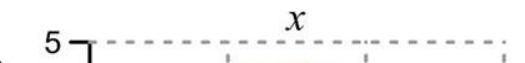

$$
\bullet \bullet \bullet y=0.25 \square \square \square y=0.5 \Delta \Delta \Delta y=0.75
$$

Figure 6. Transversal velocity component and temperature profiles for varying $\chi, R a=10^{3}$, and $T_{\mathrm{w}}=0.5$. (a) $\chi=4$; (b) $\chi=6$; (c) $\chi=4$; (d) $\chi=6$. Symbols represent values obtained with COMSOL Multiphysics (Burlington, MA).

similar, as expected from the observation of a conduction dominated regime for lower Rayleigh numbers.

Figure 6a,b confirms the observation made in the previous paragraph, with the natural convection boundary layer being responsible for steep gradients near the side wall, and a plug-type flow pattern near the vertical centerline of the SFP. Moreover, Figures $6 c, d$ are quite similar to Figure $3 \mathrm{~d}$. However, the values of the dimensionless temperature are monotonically increasing with the aspect ratio, indicating a deleterious effect this variable has on the cooling performance of the passive system.

\section{Conclusions}

Natural convection within cavities partially filled with a heat generating porous medium has been theoretically studied by applying the Generalized Integral Transform Technique (GITT). A single domain reformulation strategy was employed in combination with the GITT approach to solve the governing flow and energy equations, allowing for a hybrid numerical-analytical solution obtained with automatic error control. The single domain representation is achieved by rewriting the whole region as a Darcy model with Brinkman correction and providing space variable coefficients in the single formulation that account for the abrupt transitions between different regions. This space variable information is then carried along to the chosen eigenvalue problems, which 
form the basis of the eigenfunction expansions, and thus resulting in an excellent overall convergence behavior for both velocity and temperature fields.

This contribution has been motivated by an application dealing with wet storage of spent nuclear fuel elements, with passive cooling of the storage pool. The analysis showed that designing deeper pools and eventually the piling of the nuclear fuel elements, i.e., increasing the Rayleigh number and decreasing the aspect ratio, respectively, significantly decreases the maximum temperature within the cavity, by allowing stronger vortices to develop. Additionally, the use of heat pipes with refrigerants of lower phase change temperatures further contributes to the cooling of the cavity. The aspect ratio tends to have an opposite effect, which is attributed to the resulting larger distance between the cooling heat pipes exchangers, positioned on the sidewalls, and the center of the spent fuel elements layer. It is expected that the proposed approach shall further contribute to the thermal analysis, optimization, and control of passive cooling systems for spent nuclear fuel storage pools, summing up to the efforts on rendering nuclear facilities safer.

\section{Funding}

The authors are grateful for the financial support offered by the Brazilian Government agencies CNPq (projects no. 401237/2014-1 and no. 207750/2015-7), CAPES-INMETRO, and FAPERJ. This work was partially sponsored by ETN-Eletrobrás Termonucleares S.A., Project no. PEM-17909 with the COPPETEC Foundation.

\section{ORCID}

Renato Machado Cotta (DD http://orcid.org/0000-0003-0965-0811

\section{References}

[1] F. Arpino, G. Cortellessa, and A. Mauro, "Transient thermal analysis of natural convection in porous and partially porous cavities," Numer. Heat Transf. Part A Appl., vol. 67, no. 6, pp. 605-631, 2015. DOI: 10.1080/10407782.2014.949133.

[2] A. Tahmasebi, M. Mahdavi, and M. Ghalambaz, "Local thermal nonequilibrium conjugate natural convection heat transfer of nanofluids in a cavity partially filled with porous media using Buongiorno's model," Numer. Heat Transf. Part A Appl., vol. 73, no. 4, pp. 254-276, 2018. DOI: 10.1080/10407782.2017.1422632.

[3] G. S. Beavers and D. D. Joseph, "Boundary conditions at a naturally permeable wall," J. Fluid Mech., vol. 30, no. 01, pp. 197-207, 1967. DOI: 10.1017/S0022112067001375.

[4] D. A. Nield, "Onset of convection in a fluid layer overlying a layer of a porous medium," J. Fluid Mech., vol. 81, no. 03, pp. 513-522, 1977. DOI: 10.1017/S0022112077002195.

[5] D. Poulikakos, A. Bejan, B. Selimos, and K. R. Blake, "High Rayleigh number in a fluid overlying a porous bed," Int. J. Heat Fluid Flow, vol. 7, no. 2, pp. 109-116, 1986. DOI: 10.1016/0142-727X(86)90056-1.

[6] H. C. Brinkman, "A calculation of the viscous force exerted by a flowing fluid on a dense swarm of particles,” Appl. Sci. Res., vol. 1, no. 1, pp. 27-34, 1949. DOI: 10.1007/BF02120313.

[7] S. Whitaker, "Flow in porous media I: a theoretical derivation of Darcy's law," Transp. Porous Media, vol. 1, no. 1, pp. 3-25, 1986. DOI: 10.1007/BF01036523.

[8] J. A. Ochoa-Tapia and S. Whitaker, "Momentum transfer at the boundary between a porous medium and a homogeneous fluid-I. Theoretical development," Int. J. Heat Mass Transf., vol. 38, no. 14, pp. 2635-2646, 1995. DOI: 10.1016/0017-9310(94)00346-W.

[9] J. A. Ochoa-Tapia and S. Whitaker, "Momentum transfer at the boundary between a porous medium and a homogeneous fluid-II. Comparison with experiment," Int. J. Heat Mass Transf., vol. 38, no. 14, pp. 2647-2655, 1995. DOI: 10.1016/0017-9310(94)00347-X.

[10] G. Neale and W. Nader, "Practical significance of Brinkman extension of Darcy's law: coupled parallel flows within a channel and a boundary porous medium," Can. J. Chem. Eng., vol. 52, no. 4, pp. 475-478, 1974. DOI: $10.1002 /$ cjce.5450520407.

[11] R. M. Cotta, Integral Transforms in Computational Heat and Fluid Flow, 1st ed. Boca Raton, FL: CRC Press, 1993. 
[12] R. M. Cotta and M. D. Mikhailov, Heat Conduction: Lumped Analysis, Integral Transforms, Symbolic Computation, 1st ed., Chichester, UK: Wiley Interscience, 1997.

[13] R. M. Cotta, The Integral Transform Method in Thermal, Fluid Sciences and Engineering, 1st ed., New York, NY: Begell House, 1998.

[14] R. M. Cotta and M. D. Mikhailov, "Hybrid methods and symbolic computations," in Handbook of Numerical Heat Transfer, 2nd ed., Chapter 16, W. J. Minkowycz, E. M. Sparrow, and J. Y. Murthy, Eds. New York: John Wiley, 2006, pp. 493-522.

[15] R. M. Cotta, D. C. Knupp, and C. P. Naveira-Cotta, Analytical Heat and Fluid Flow in Microchannels and Microsystems, 1st ed. New York, NY: Springer, 2016.

[16] R. M. Cotta, D. C. Knupp, and J. N. N. Quaresma, "Analytical methods in heat transfer," in Handbook of Thermal Science and Engineering, 1st ed., Chapter 1, Francis A. Kulacki, Eds. Switzerland: Springer International Publishing, 2017. DOI: 10.1007/978-3-319-32003-8_2-1

[17] M. A. Leal, J. S. Perez Guerrero, and R. M. Cotta, "Natural convection inside two-dimensional cavities: the integral transform method," Commun. Numer. Meth. Eng., vol. 15, no. 2, pp. 113-125, 1999.

[18] M. A. Leal, H. A. Machado, and R. M. Cotta, "Integral transform solutions of transient natural convection in enclosures with variable fluid properties," Int. J. Heat Mass Transfer, vol. 43, no. 21, pp. 3977-3990, 2000. DOI: 10.1016/S0017-9310(00)00023-5.

[19] C. F. T. Matt, J. N. N. Quaresma, and R. M. Cotta, “Analysis of magnetohydrodynamic natural convection in closed cavities through integral transforms," Int. J. Heat Mass Transf, vol. 113, pp. 502-513, 2017. DOI: 10.1016/j.ijheatmasstransfer.2017.05.043.

[20] S. C. Hirata, B. Goyeau, D. Gobin, M. Carr, and R. M. Cotta, "Linear stability of natural convection in superposed fluid and porous layers: influence of the interfacial modelling," Int. J. Heat Mass Transf, vol. 50, no. 7-8, pp. 1356-1367, 2007. DOI: 10.1016/j.ijheatmasstransfer.2006.09.038.

[21] S. C. Hirata, B. Goyeau, D. Gobin, M. Chandesris, and D. Jamet, "Stability of natural convection in superposed fluid and porous layers: Equivalence of the one- and two-domain approaches," Int. J. Heat Mass Transf, vol. 52, no. 1-2, pp. 533-536, 2009. DOI: 10.1016/j.ijheatmasstransfer.2008.07.045.

[22] C. Baohua and R. M. Cotta, "Integral transform analysis of natural convection in porous enclosures," Int. J. Num. Meth. Fluids, vol. 17, no. 9, pp. 787-801, 1993.

[23] L. S. De. B. Alves and R. M. Cotta, "Transient natural convection inside porous cavities: hybrid numericalanalytical solution and mixed symbolic-numerical computation," Num. Heat Transfer, Part A-Appl., vol. 38 , no. 1, pp. 89-110, 2000.

[24] H. Luz Neto, J. N. N. Quaresma, and R. M. Cotta, "Natural convection in three-dimensional porous cavities: integral transform method," Int. J. Heat Mass Transfer, vol. 45, no. 14, pp. 3013-3032, 2002.

[25] G. G. C. Lima, C. A. C. Santos, A. Haag, and R. M. Cotta, "Integral transform solution of internal flow problems based on Navier-Stokes Equations and primitive variables formulation," Int. J. Num. Meth. Eng, vol. 69, no. 3, pp. 544-561, 2007. DOI: 10.1002/nme.1780.

[26] J. S. Perez-Guerrero and R. M. Cotta, "Integral transform solution for the lid-driven cavity flow problem in streamfunction-only formulation," Int. J. Num. Meth. Fluids, vol. 15, no. 4, pp. 399-409, 1992. DOI: 10.1002/fld.1650150403.

[27] J. S. Perez-Guerrero and R. M. Cotta, "Benchmark integral transform results for flow over a backward-facing step," Comput. Fluids, vol. 25, no. 5, pp. 527-540, 1996. DOI: 10.1016/0045-7930(96)00005-9.

[28] J. S. Perez-Guerrero, J. N. N. Quaresma, and R. M. Cotta, "Simulation of laminar flow inside ducts of irregular geometry using integral transforms," Comput. Mech., vol. 25, no. 4, pp. 413-420, 2000. DOI: 10.1007/s004660050488.

[29] R. Ramos, J. S. Perez-Guerrero, and R. M. Cotta, "Stratified flow over a backward facing step: hybrid solution by integral transforms," Int. J. Num. Meth. Fluids, vol. 35, no. 2, pp. 173-197, 2001.

[30] C. A. M. Silva, E. N. Macedo, J. N. N. Quaresma, L. M. Pereira, and R. M. Cotta, "Integral transform solution of the Navier-Stokes equations in full cylindrical regions with streamfunction formulation," Int. J. Num. Meth. Biomedical Eng., vol. 26, no. 11, pp. 1417-1434, 2010.

[31] D. C. Knupp, R. M. Cotta, and C. P. Naveira-Cotta, "Heat transfer in microchannels with upstream-downstream regions coupling and wall conjugation effects," Numer. Heat Transf. Part B Fundam., vol. 64, no. 5, pp. 365-387, 2013. DOI: 10.1080/10407790.2013.810535.

[32] D. C. Knupp, C. P. Naveira-Cotta, and R. M. Cotta, "Conjugated convection-conduction analysis in microchannels with axial diffusion effects and a single domain formulation,” J. Heat Transfer, vol. 135, no. 9, pp. 091401, 2013. DOI: $10.1115 / 1.4024425$.

[33] D. C. Knupp, C. P. Naveira-Cotta, and R. M. Cotta, "Theoretical-experimental analysis of conjugated heat transfer in nanocomposite heat spreaders with multiple microchannels," Int. J. Heat Mass Transf., vol. 74, pp. 306-318, 2014. DOI: 10.1016/j.ijheatmasstransfer.2014.03.005. 
[34] D. C. Knupp, R. M. Cotta, C. P. Naveira-Cotta, and S. Kakaç, “Transient conjugated heat transfer in microchannels: Integral transforms with single domain formulation,” Int. J. Therm. Sci., vol. 88, pp. 248-257, 2015. DOI: 10.1016/j.ijthermalsci.2014.04.017.

[35] D. C. Knupp, R. M. Cotta, and C. P. Naveira-Cotta, "Fluid flow and conjugated heat transfer in arbitrarily shaped channels via single domain formulation and integral transforms," Int. J. Heat Mass Transf., vol. 82, pp. 479-489, 2015. DOI: 10.1016/j.ijheatmasstransfer.2014. 11. 007.

[36] J. R. B. Souza et al., "Thermal analysis of anti-icing systems in aeronautical velocity sensors and structures," J Braz. Soc. Mech. Sci. Eng., vol. 38, no. 5, pp. 1489-1509, 2016. DOI: 10.1007/s40430-015-0449-7.

[37] R. M. Cotta, C. P. Naveira-Cotta, D. C. Knupp, J. L. Z. Zotin, P. C. Pontes, and A. P. Almeida, "Recent advances in computational-analytical integral transforms for convection-diffusion problems," Heat Mass Transfer, vol. 54, pp. 2475-2496, 2018. (Invited Paper). DOI: 10.1007/s00231-017-2186-1.

[38] K. M. Lisboa and R. M. Cotta, "Hybrid integral transforms for flow development in ducts partially filled with porous media," Proc. R. Soc. A Math. Phys. Eng. Sci., vol. 474, no. 2209, p. 20170637, 2018. DOI: 10.1098/rspa.2017.0637.

[39] K. M. Lisboa and R. M. Cotta, "On the mass transport in membraneless flow batteries with flow-by configuration," Int. J. Heat Mass Transf., vol. 122, pp. 954-966, 2018. DOI: 10.1016/ j.ijheatmasstransfer.2018.02.002.

[40] M. Wang, H. Zhao, Y. Zhang, G. Su, W. Tian, and S. Qiu, "Research on the designed emergency passive residual heat removal system during the station blackout scenario for CPR1000," Ann. Nucl. Energy, vol. 45, pp. 86-93, 2012. DOI: 10.1016/j.anucene.2012.03.004.

[41] A. Kaliatka, V. Ognerubov, and V. Vileiniskis, "Analysis of the processes in spent fuel pools of ignalina NPP in case of loss of heat removal," Nucl. Eng. Des., vol. 240, no. 5, pp. 1073-1082, 2010. DOI: 10.1016/ j.nucengdes.2009.12.026.

[42] T. C. Hung, V. K. Dhir, B. S. Pei, Y. S. Chen, and F. P. Tsai, "The development of a three-dimensional transient CFD model for predicting cooling ability of spent fuel pools," Appl. Therm. Eng., vol. 50, no. 1, pp. 496-504, 2013. DOI: 10.1016/j.applthermaleng. 2012.06.042.

[43] C. Ye, M. G. Zheng, M. L. Wang, R. H. Zhang, and Z. Q. Xiong, "The design and simulation of a new spent fuel pool passive cooling system," Ann. Nucl. Energy, vol. 58, pp. 124-131, 2013. DOI: 10.1016/ j.anucene.2013.03.007.

[44] L. Lima, N. Mangiavacchi, and L. Ferrari, "Stability analysis of passive cooling systems for nuclear spent fuel Pool,” J. Braz. Soc. Mech. Sci. Eng., vol. 39, no. 3, pp. 1019-1031, 2017. DOI: 10.1007/s40430-0160589-4.

[45] U. Appenzeller, External spent fuel storage facility at the nuclear power plant in Gösgen, IAEA Technical Meeting on SNF storage options, 2-4 July 2013, Vienna, Austria. 